\title{
Monitoring of Dissolving Processes of Amphipathic Molecules from Aqueous Surface by Laser-Induced Two-Photon Ionization
}

\section{Hirofumi KA WAZUMI, Takeshi KAIEDA, Kohshin OHMUKAI, Miki SATO*, Takanori INOUE*, Teiichiro OGAWA*}

Department of Industrial Chemistry, Kinki University in Kyushu, Iizuka 820, Japan

*Department of Molecular Science and Technology, Kyushu University, Kasuga 816, Japan

\begin{abstract}
Laser two-photon ionization technique has been applied to monitor dissolving processes of probe molecules from solution surfaces. The time courses of the photoionization charge decay were measured by changing the properties of the bulk phase after the addition of the amphipathic molecules. The dissolving rate of pyrenebutyric acid, aminopyrene and pyrenesulfonic acid was strongly depended on $\mathrm{pH}$ in the bulk solution. The dissociation equilibrium of the conjugated acid-base system governed the solubility of the amphipathic molecules. Effects of surfactants were also observed in the dissolving process of pyrenedecanoic acid.
\end{abstract}

Keywords Photoionization, air-liquid interface, probe molecule, surface solubilization

The air-liquid interface, that is the solution surface, plays important roles in a variety of technologies such as detergency, coating, wetting, adhesion. 1 Microscopic phenomena at the surface have to be elucidated for not only the technologies but also the physical chemistry. For example, the elementary protocol includes the measurement of surfacemodified $\mathrm{pH}$ and dielectric constant, and the monitoring of surfactant adsorption and surface solubilization. A few analytical techniques for these research field have been reported. Interfacial tension method can be used to determine the adsorbed amount of the surfactant. 2 Radiotracer method has been useful in monitoring the adsorption dynamics. 3,4 Neutron reflectivity measurements 5 and second harmonic generation $\mathbf{6}$ are employed to study the structure of surface monolayers and aggregates. Second harmonic generation is also used to estimate modified pKa of an alkyl aniline at the surface. ${ }^{7}$

Recently, the laser-induced multi-photon ionization (MPI) has been reported as a new versatile technique for the solid and liquid surface studies.8-12 A laser irradiation on the aqueous surface can produce a multi-photon ionization of photoabsorbing molecules. An ejected electron from the molecules that exist on or near the surface is measured efficiently by using an electrode above the surface. The ions and electrons produced in the bulk phase are solvated by surrounding water molecules to create the ion-electron pair and recombine. This recombination occurs in a very short distance, which is in the scale of Onsager's distance and is less than a few $\mathrm{nm}$. Therefore, the MPI signal is very surface selective and can be used for the analysis of the in situ surface. 11 The concentration and mass sensitivity are enough to detect the surface molecules. Furthermore, the MPI technique provides the spectral information to scan the wavelength of the excitation laser. 12

In this paper, the MPI technique has been applied to monitor dissolving processes of amphipathic molecules from the air-liquid interfaces. Time courses of the MPI signal have been measured after the expansion of the photoabsorbing amphipathic molecules on the solution surfaces. The decrease of the signal corresponds to the dissolving of the probe molecules from the surface into the bulk phase. Effects of $\mathrm{pH}$ of the bulk solution were observed in the time course measurements of surface MPI signal. Effects of the addition of surfactants to the water were also observed for the amphipathic molecule with a long alkyl chain. The dissolving dynamics has been discussed from these results.

\section{Experimental}

The experimental apparatus was described in 


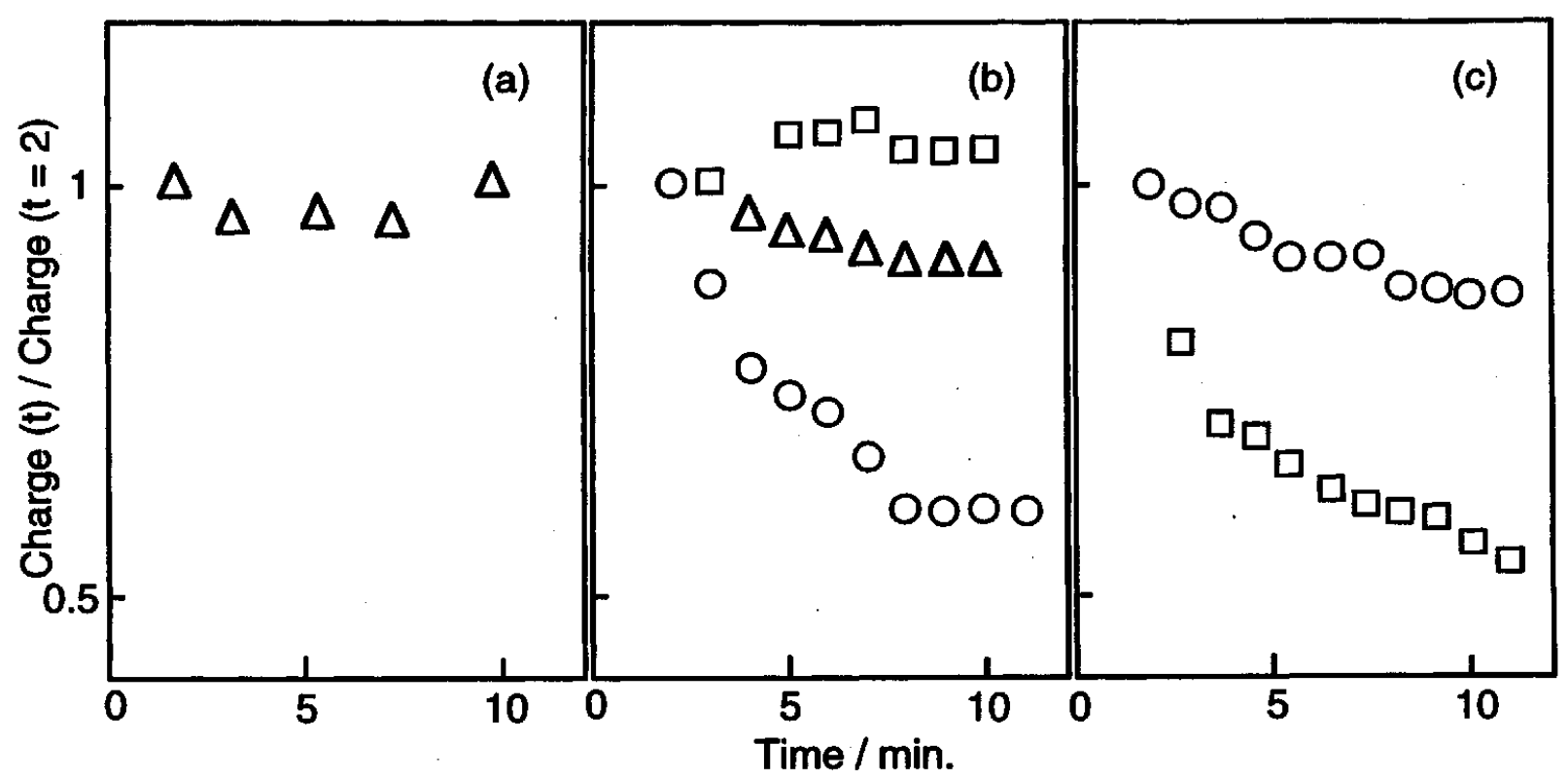

Fig. 1 Effects of $\mathrm{pH}$ on the time course of the surface MPI signal decay. $\square ; \mathrm{pH} 2.8, \Delta ; \mathrm{pH} 5.0, \mathrm{O} ; \mathrm{pH} 12$. (a); sodium 1-pyrene-sulfonate/PySA, (b); 1-Pyrenebutyric acid/PyBA, (c); 1-aminopyrene / PyAm. The concentration of the probe molecules is $1.0 \times 10^{-7} \mathrm{~mol} / \mathrm{dm}^{3}$.

detail previously.11 A nitrogen laser (UV-24, Molectron; $337 \mathrm{~nm}, 10-\mathrm{ns}$ duration, $10 \mathrm{~Hz}$ ) was focused softly with a quartz lens $(f=900 \mathrm{~mm}$ ) on the solution surface at the incident angle of 85 degree. Typical laser energy was $1.0 \mathrm{~mJ}$. The laser irradiation was blocked except for the short measuring time of a few second to prevent photodecomposition of the probe molecules. A stainless steel cell with a solution reservoir $(0.5 \mathrm{ml}$, surface area $1.0 \mathrm{~cm}^{2} \times$ depth $0.5 \mathrm{~cm}$ ) worked as a negative and grounded electrode and was connected to a current amplifier (427, Keithlay). A circular positive electrode was located $8 \mathrm{~mm}$ above the aqueous surface and the applied voltage was $1.5 \mathrm{kV}$. The photoionization charge was obtained by integrating the transient photocurrent measured with a digital storagescope (DS-6411, Iwatsu).

1-Pyrenebutyric acid (PyBA, Wako), 1-aminopyrene (PyAm, Wako), sodium 1-pyrenesulfonate (PySA, Molecular Probes), 10-(1-pyrene)decanoic acid (PyDA, Sigma), sodium n-dodecylbenzenesulfonate (SDBS, Wako) and cetyltrimethylammonium bromide (CTAB, Wako) were used as obtained. The water was distilled, deionized, filtered, and distilled in a quartz vessel. For the $\mathrm{pH}$ adjustment, the aqueous phase consisted of $2.5 \times 10^{-2}$ $\mathrm{mol} / \mathrm{dm}^{3}$ sodium phosphate buffer. In sample preparation, the water or buffer solution was placed in the reservoir at first. $10 \mu$ l-benzene solution containing the probe molecules was spreaded on the solution by a microsyringe, and the solvent was vaporized. The MPI measurement was started within 2 minutes after the addition of the probe molecules.

\section{Results and Discussion}

\section{Effect of $p H$}

All probe molecules provided the MPI signal from the aqueous surface. Their photoionization charges were quadratically proportional to the laser pulse energy. The probe molecules with a pyrene moiety show a large absorption at the laser wavelength and should ionize via a stepwise two-photon process. 10 The photoionization charge of PyBA was linearly proportional to the amount of the molecule spreaded on the surface between $1.0 \times 10^{-6} \mathrm{~mol}$ and $1.0 \times 10^{-9}$ mol. The latter value corresponds a part per five hundred of the monolayer, in which a molecular area was estimated to be $0.34 \mathrm{~nm}^{2}$ according to a surface pressure measurement and a space-filling molecular modeling. 13 In the following measurements the amounts of the probe molecules were set to be $1.0 \mathrm{x}$ $10^{-7} \mathrm{~mol}$.

A transient photocurrent had the pulse width of about $500 \mu$ s under the present experimental condi- 


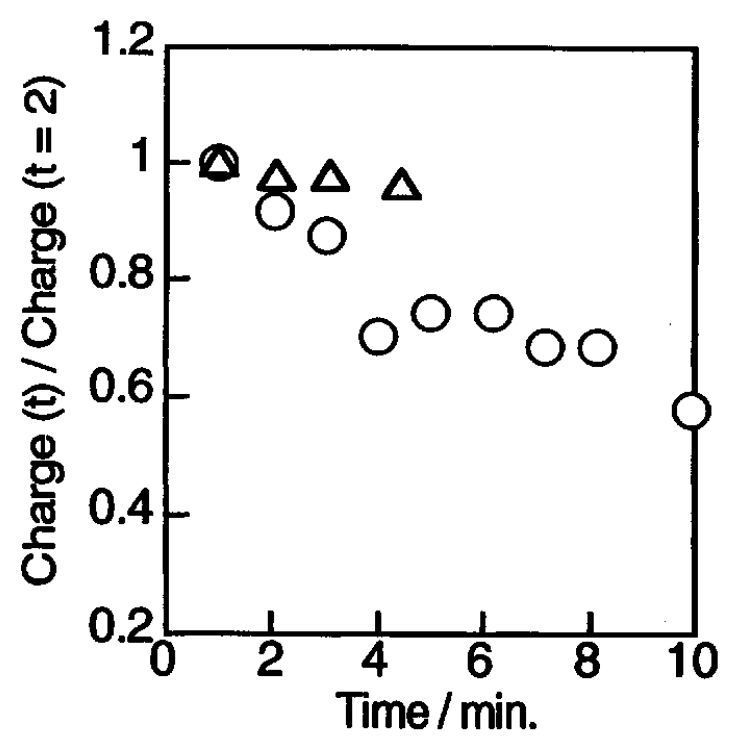

Fig. 2 Time courses of the MPI signal from PyDA in the addition of surfactants. ( $O$ ); SDBS,

$(\Delta) ; C T M B$. The concentration of the surfactants is 1.0 $\times 10^{-5} \mathrm{~mol} / \mathrm{dm}^{3}$.

tion. The photocurrent intensity decreased gradually in several tens minutes after the addition of the probe molecules except that of PySA, as shown in Fig. 1. This decay should be due to the dissolving process of the amphipathic molecules from the surface into the bulk phase. The decay curves were normalized to the value at 2 minutes after the sample preparation. The decay rates strongly depend on the $\mathrm{pH}$ and the amphipathic molecule. For PySA in Fig. 1-(a), no decay was observed and the $\mathrm{pH}$ effect was also not observed. Since PySA has a strong acidic moiety of a sulfonic group and completely dissociates into the ion at $\mathrm{pH}$ used, the water solubility of PySA is very large. Therefore, the dissolving process from the surface is very fast and the partition between the surface and the bulk phase attains equilibrium within 1 minute, which is before the first measurement. The dissolving process of the ionic species from the surface is fast, as shown in the case of PySA. Therefore, the slow decays of the amphipathic molecules such as PyBA and PyAm indicate the existence of the energy barrier in the diffusion into the aqueous phase, that is the adsorption energy to the surface.

The decay rate for PyBA in Fig. 1-(b) increases with increasing the pH. PyBA has a weak acidic moiety of a carboxyl group. At the high pH 12, nearly all PyBA molecules dissociate into the ions and quickly dissolve into the bulk phase; only a part per $10^{12}-4.6=10^{7.4}$ of PyBA may be protonated if

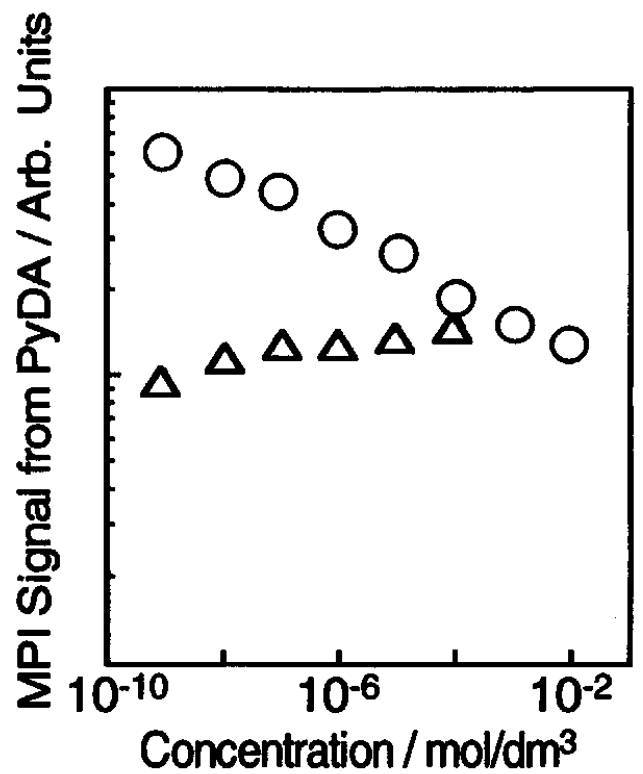

Fig. 3 Dependence of the surfactant concentration on the MPI signal from PyDA. ( $)$; SDBS,

$(\Delta)$; CTMB. The concentration of the surfactants is $1.0 \times 10^{-5} \mathrm{~mol} / \mathrm{dm}^{3}$.

the dissociation constant of carboxyl group in PyBA is the same as that in acetic acid, $\mathrm{pKa}=4.6 .14$ Lowering the $\mathrm{pH}$, the protonated PyBA molecules increase and the overall dissolving process decelerates. The slight increase at $\mathrm{pH} 2.8$ may be due to the cluster formation upon the laser irradiation because of the insoluble character of non-ionic PyBA. PyAm shows the opposite behavior because it has a weak basic moiety of an amino group. The dissociation equilibrium of the conjugated acid-base system should govern the solubility of the amphipathic molecules from the surface. A precise measurement of the $\mathrm{pH}$ effect could reveal the "effective" pH and pKa at the aqueous surface.7,15

\section{Effect of Surfactants}

The effects of the addition of the surfactants to the water have been studied by using the surface MPI signal from PyDA. Figure 2 presents the time courses of the signal decays in the case of SDBS and CTMB; their concentrations in the solution are $1.0 \mathrm{x}$ $10^{-5} \mathrm{~mol} / \mathrm{dm}^{3}$. The addition of the anionic surfactant of SDBS indicates the steady decay that is similar to

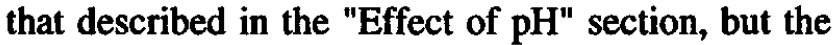
addition of the cationic one does not. The dependence of the surfactant concentration was observed, as shown in Fig. 3. We measured the signal intensities 10 minutes later after the sample preparation, when the signals became constant. With increasing the SDBS and CTAB concentration, the 
surface MPI signal gradually decreases and slightly increases, respectively. The concentration of the surfactants in Fig. 3 is lower than its critical micelle concentration $(\mathrm{cmc})$. We conclude that a small number of the surfactants and the probe molecules forms into surface micelles that is small pre-micelles in the two-dimensional surface. 16,17 The results in the dependences of the time and concentration seem to be strange comparing to the simple estimation from the electrostatic interaction, where the cationic micelle of CTAB solubilizes more anionic probe molecules than the anionic micelle of SDBS does. Taking into account of the large insolubility of PyDA, the small assemble with opposite charges, CTAB and PyDA, may be reduced the solubility of the surface micelle into the bulk phase.

The laser-induced MPI technique has been successfully applied to monitor dissolving processes at the air-liquid interfaces. The time course measurements of the surface MPI signal decay directly illustrate the dissolving processes from the surface. The surface selectivity and the high sensitivity of the surface MPI are useful for studying the dynamics at the air-liquid interfaces and offer a great potential to the further applications.

\section{References}

1. R. Sharma, "Surfactant Adsorption and Surface Solubilization", American Chenical Society, Washington, DC, 1995.

2. A. W. Adamson, "Physical Chemistry of Surfaces 5th Edition", Wiley-Interscience, New York, NY, 1990.

3. R. Matuura, H. Kimizuka, S. Miyamoto, R. Shimozawa and K. Yatsunami, Bull. Chem. Soc. Jpn., 32, 404 (1959).

4. G. G. Jayson and G. J. Thomson, J. Colloid Interface Sci., 111, 65 (1986).

5. I. R. Gentle, P. M. Saxille, J. W. White and J. Penfold, Langmuir, 9, 646 (1993).

6. G. S. Frysinger, A. A. Barnoski and G. L. J. Gaines, Langmuir, 10, 2277 (1994).

7. X. Zhao, S. Subrahmanyan and K. B. Eisenthal, Chem. Phys. Letters, 171, 558 (1990).

8. T. Ogawa, T. Yasuda and H. Kawazumi, Anal. Chem., 64, 2615 (1992).

9. H. Kawazumi, T. Yasuda and T. Ogawa, Anal. Chim. Acta, 283, 111 (1993).

10. H. Chen, T. Inoue and T. Ogawa, Anal. Chem., 66, 4150 (1994).

11. T. Inoue, K. Masuda, K. Nakashima and T.
Ogawa, Anal. Chem., 66, 1012 (1994).

12. T. Ogawa, H. Chen, T. Inoue and $K$. Nakashima, Chem. Phys. Letters, 229, 328 (1994).

13. T. Yamazaki, N. Tamai and I. Yamazaki, Chem. Phys. Letters, 124, 326 (1986).

14. R.C.Weast ed., "CRC Handbook of Chemistry and Physics 78rd Edition", CRC Press, Boca Raton, FL (1997).

15. Y. R. Shen, Ann. Rev. Phys. Chem., 43, 627 (1992).

16. J. Israelachvili, Langmuir, 10, 3774 (1994).

17. J. Leimbach, J. Sigg and H. Rupprecht, Colloids and Surfaces, 94, 1 (1995). 